\title{
El estudio de caso como estrategia de aprendizaje en estudiantes de nutrición y dietética
}

\author{
Claudia A. TRONCOSO-PANTOJA, Gina A. BURDILES-FERNÁNDEZ, Mauricio A. SOTOMAYOR-CASTRO, \\ Lorena F. ECHEVERRÍA-SÁNCHEZ
}

Introducción. La formación en el ámbito de la salud debe considerar diversas estrategias pedagógicas que favorezcan el desarrollo de competencias profesionales y la manera en que sus resultados se comunican científicamente.

Objetivo. Explorar el uso del estudio de caso como estrategia de formación profesional y académica en estudiantes de la carrera de Nutrición y Dietética.

Sujetos y métodos. Se implementó el estudio de caso como intervención didáctica para el aprendizaje activo. Se formaron equipos de trabajo, que debieron planificar e implementar una intervención nutricional según una estructura programática entregada por docentes. Una vez finalizada la actividad, se realizó un escrito con formato científico que incluyó el uso de bases de datos para los aspectos teóricos del artículo, además de la presentación de la experiencia en un seminario público. Se evalúa la actividad desarrollada a través de cuestionario de respuestas abiertas al inicio y a la finalización del proceso.

Resultados. Los estudiantes mencionan que la estrategia fue una instancia que sirvió para fortalecer competencias en el manejo nutricional de adultos mayores y para reforzar habilidades de redacción y vocabulario pertinentes en su desarrollo disciplinar. Como limitación, se identifica la elaboración de la discusión en el documento escrito.

Conclusiones. El trabajo con estudio de caso es una estrategia de aprendizaje integral que faculta el manejo terapéutico de una persona atendida, el desarrollo de competencias profesionales de redacción científica y la difusión académica en formato oral.

Palabras clave. Aprendizaje activo. Comunicación científica. Estrategia pedagógica. Estudio de caso.

\section{Study case as a learning strategy for students of Nutrition and Dietetics}

Introduction. Training in the healthcare area must consider several pedagogical strategies that favor the development of competences as skills to scientifically communicate the results of a professional chores.

Aim. To explore the use of study case as a professional and academic learning strategy in students of the Nutrition and Dietetics career.

Subjects and methods. The study case was implemented as a didactic intervention for active learning. In working teams, the students had to plan and implement a nutritional intervention based on a programmatic structure given by the professors. Once the activity ended, they made a scientific format writing, including the use of databases for the theoretical aspects of the article, besides the presentation of the experience in a public seminar. This research asset the developed activity through survey of open answers applied to the students at the beginning and the end of the process.

Results. According to the students, the strategy was an instance that helped to strengthen some competence in the nutritional handling of elder people, besides reinforcing writing skills and vocabulary related to their disciplinary development. As a preset limit, it has been identified the elaboration in the writing document of the discussion.

Conclusions. The workshop with a study case proved to be an integral learning strategy that, for one side, faculties the therapeutically management of a patient, the development of professional competences in the scientific writing development, besides allowing academic diffusion through the oral presentations of their results.

Key words. Active learning. Pedagogical strategy. Scientific communication. Study case.
Departamento de Ciencias Clínicas y Preclínicas; Facultad de Medicina (C.A. Troncoso-Pantoja, M.A. Sotomayor-Castro). Departamento de Ciencias del Lenguaje y Literatura; Facultad de Educación (G.A. BurdilesFernández). Centro de Investigación en Educación y Desarrollo (C.A. Troncoso-Pantoja, G.A. BurdilesFernández). Departamento de Bioética (L.F. Echeverría-Sánchez). Universidad Católica de la Santísima Concepción. Concepción, Chile.

Correspondencia:

Profa. Claudia Andrea Troncoso Pantoja. Facultad de Medicina. Universidad Católica de la Santísima Concepción. Campus San Andrés. Alonso de Ribera, 2850. Concepción, Chile.

E-mail:

ctroncosop@ucsc.cl

Financiación:

Proyecto CIEDE 02-2017, Centro de Investigación en Educación y Desarrollo (CIEDE-UCSC), Universidad Católica de la Santísima Concepción. Proyecto Fondecyt Iniciación 11160863.

Recibido: 13.09.18.

Aceptado: 11.10.18.

Conflicto de intereses: No declarado.

Competing interests: None declared.

(c) 2019 FEM

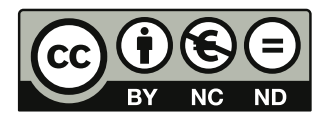

Artículo open access bajo la licencia CC BY-NC-ND (https:// creativecommons.org/licenses/ by-nc-nd/4.0/).

ISSN: 2014-9832

ISSN (ed. digital): 2014-9840 


\section{Introducción}

El caso clínico, reporte de caso o estudio de caso, según el contexto geográfico, corresponde a un tipo de publicación biomédica de gran importancia para todas las disciplinas del área de la salud [1] debido a su valor educativo, clínico y científico [2]. Entre sus fortalezas, destaca que facilita la comprensión, razonamiento y manejo de la incertidumbre en la práctica clínica [3] y permite disponer de información para evaluar la mejor manera de realizar una intervención terapéutica o de promoción en salud, a fin de mejorar la calidad de vida de una persona [4]. Por otra parte, como limitaciones, se menciona que ocupa el nivel más bajo de la medicina basada en la evidencia [5], que no es extrapolable y que sólo describe un caso o evento clínico [6].

Siguiendo el rigor científico y según recomendaciones, entre otras, del grupo CARE [7], el estudio de caso presenta una estructura y estilo para su elaboración que, en una parte preliminar, incorpora el título, autoría y resumen del caso; luego presenta el cuerpo del artículo, con su introducción, presentación del caso y discusión; finalmente, incluye citas bibliográficas, agradecimientos o anexos, si fuesen necesarios [8]. El estudio de caso, cuya presencia es considerable en publicaciones seriadas [9], aparece en secciones exclusivas de presentación de casos [10] y, además de facilitar la difusión académica, posee finalidades educativas, especialmente orientadas a estudiantes de pregrado o de formación en al área médica [11].

En el desarrollo de la formación profesional universitaria se requieren estrategias didácticas que permitan un aprendizaje activo de los estudiantes, como juegos de rol, debates, seminarios o proyectos de investigación [12]. En la actualidad, la formación profesional no sólo exige que los estudiantes adquieran conocimientos, sino que también desarrollen competencias que les permitan solucionar de forma creativa y autónoma problemas propios de su quehacer profesional [13]. Esto implica un desafío para los estudiantes y para los docentes, quienes deben integrar nuevas herramientas de estudio [14] y atender a los diferentes estilos de aprendizaje [15].

Las estrategias de aprendizaje son representaciones mentales resultantes de un análisis reflexivo retratado en un planteamiento de acciones que permiten mejorar el conocimiento a través de la toma de decisiones [16]. Algunas técnicas utilizadas frecuentemente en este ámbito corresponden a nemotecnias, subrayado, parafraseo, mapas conceptuales $\mathrm{o}$ autocuestionarios, aunque lo esencial no reside en la técnica propiamente dicha, sino en la manera en que se ejecutan estas técnicas y en la finalidad perseguida por ellas $[17,18]$. De hecho, existe relación entre la carrera cursada en la educación terciaria y los estilos de aprendizaje, que se modifican con el avance en el respectivo plan de estudios, respondiendo a una adaptación cognitiva a la vida universitaria y a las estrategias que utilizan sus educadores [19]. Sin embargo, en el desarrollo de la vida universitaria, se han observado algunos problemas en el momento de ejecutar las estrategias de aprendizaje, como los relacionados con la motivación, los conocimientos previos requeridos y los criterios de evaluación o autorregulación, que constituyen inconvenientes en la formación profesional, condicionan el desempeño académico de los estudiantes [20] $y$, de algún modo, favorecen su vulnerabilidad.

El panorama descrito motiva esta propuesta didáctica, la cual se aproxima, en el proceso de profesionalización de los estudiantes, al desarrollo de adecuadas prácticas centradas en sus necesidades.

Se propone indagar en estrategias de aprendizaje y de organización que permitan, por una parte, reforzar la formación disciplinar de los estudiantes en el manejo dietético-terapéutico y nutricional de un grupo de edad específico, en este caso, adultos mayores, y además potenciar la formación académica a través del dominio de discurso especializado mediante el uso del estudio de caso.

El objetivo de esta intervención es explorar el uso del estudio de caso como estrategia de formación profesional y académica en estudiantes de la carrera de Nutrición y Dietética para el manejo dietético-terapéutico de adultos mayores durante el primer semestre del año 2017. Esta meta se alcanza mediante la promoción del aprendizaje activo de un grupo estudiantes de la carrera y se concreta en la implementación de un plan de manejo dietéticoterapéutico en adultos mayores, el desarrollo de las competencias necesarias para elaborar un estudio de caso a partir de los resultados del plan de alimentación desarrollado en el curso y, finalmente, el análisis de las percepciones de los alumnos participantes acerca del estudio de caso como estrategia de aprendizaje en su formación profesional y académica.

\section{Sujetos y métodos}

Los estudiantes formaron equipos de trabajo que, de manera autónoma, identificaron a una persona mayor que cumpliera con los criterios de selección considerados para esta actividad. Los estudiantes 
planificaron e implementaron una intervención terapéutica a través de la dieta, que se entregó a la persona beneficiada mediante sesiones educativas en su domicilio. Una vez finalizada la intervención, los estudiantes elaboraron un escrito con formato científico establecido (estudio de caso) y programaron y realizaron una presentación académica oral.

La aplicación de la estrategia educativa se realizó en siete fases. Todas ellas se desarrollaron dentro de la actividad curricular denominada 'Manejo nutricional de la persona mayor', que responde a la certificación académica de Gerontología Nutricional, impartida en el primer semestre del cuarto nivel del plan de estudios de la carrera de Nutrición y Dietética de la Universidad Católica de la Santísima Concepción (UCSC), en Chile.

\section{Fase 1: identificación de la necesidad de implementación de la estrategia educativa}

El planteamiento de esta táctica educativa surge de la reflexión interdisciplinaria entre docentes de la carrera ya mencionada y un académico especialista en lingüística de la misma institución, con investigación sobre el género discursivo de un estudio de caso en medicina [21]. Se ha observado que los estudiantes de la carrera de Nutrición y Dietética de la UCSC precisan un reforzamiento en actividades académicas relacionadas con la organización individual y colectiva de sus métodos de estudio, como es la búsqueda de fuentes científicas, lectura y escritura, por lo que se reflexionó sobre la necesidad de proponer una estrategia didáctica, como el estudio de caso, para desarrollar habilidades propias del quehacer profesional y para la comunicación científica en un profesional del área de salud.

\section{Fase 2: diseño de la intervención}

Los docentes diseñaron material escrito destinado a informar a los estudiantes sobre la actividad a realizar, incluyendo una descripción del estudio de caso y las acciones a desarrollar. De este modo se incorporaron los siguientes temas:

- Definición del estudio de caso.

- Aplicación del estudio de caso para la certificación académica de Gerontología Nutricional.

- Tributación de la actividad a competencias genéricas y específicas del plan de estudios de la carrera.

- Estructura del informe escrito del estudio de caso.

El escrito se complementaba con sesiones teóricas desarrolladas por docentes a cargo del proyecto.

\section{Fase 3: implementación}

Esta fase se inicia con el diagnóstico, consistente en un cuestionario de preguntas abiertas destinado a conocer lo que los estudiantes saben acerca del estudio de caso como fuente de información académica y disciplinar:

- ¿Qué sabe acerca de los estudios de caso como artículo científico?

- ¿Cuántas veces ha leído un estudio de caso? Si su respuesta es negativa, ¿por qué?

- ¿Qué complejidad cree que tendrá el desarrollo de su estudio de caso? ¿Por qué?

- ¿Cree que el estudio de caso le permitirá adquirir nuevas habilidades? ¿Por qué?

Una vez presentados los conceptos generales de la estrategia educativa, se solicitó a los estudiantes que formaran equipos de trabajo compuestos por dos o tres alumnos. La instrucción de trabajo indicaba que el equipo debía identificar de forma autónoma a un adulto mayor que cumpliera con las siguientes características:

- Persona mayor de 60 años.

- Con presencia de patología que requiriera manejo nutricional.

- Disponibilidad para participar en la intervención.

- Firma del consentimiento informado, en donde el paciente identificara su voluntad de colaborar en la intervención realizada, con el compromiso de respetar su privacidad y permitirle abandonar, sin coerción, la actividad.

Tras la búsqueda del caso y la firma del consentimiento informado, los estudiantes realizaron un diagnóstico que les permitió formular e implementar un plan de trabajo dietético-terapéutico de al menos cuatro semanas para la atención directa de la persona beneficiada en su domicilio.

\section{Fase 4: estrategias evaluativas del proceso}

Para el seguimiento de la actividad, se realizaron dos evaluaciones de proceso consistentes en sendas presentaciones orales sobre los avances del trabajo:

- Avance 1: presentación de los antecedentes generales del sujeto de estudio, el objetivo de la intervención, la determinación y justificación de la intervención o del procedimiento dietético nutricional a desarrollar, y los mecanismos que permiten su seguimiento.

- Avance 2: incorporación de los antecedentes de la persona, las metas de la intervención y los resultados de esta última. 
Los avances 1 y 2 fueron evaluados por el cuerpo docente a través de pautas de evaluación previamente compartidas con los estudiantes.

\section{Fase 5: elaboración del caso clínico}

Una vez desarrollada la intervención, a fin de guiar la escritura del informe del estudio de caso, los estudiantes recibieron una plantilla que debía completarse y que incluía instrucciones para su realización y registro de los siguientes datos:

- Título: máximo 15 palabras.

- Autores: nombre y apellidos, con un número entre paréntesis para identificar la filiación del autor.

- Resumen: máximo 250 palabras. Debe incluir: breve introducción, objetivo de la intervención, sujetos y métodos, resultados y conclusiones.

- Palabras clave: entre tres y cinco.

- Introducción: resumen de los antecedentes teóricos que contextualizan la intervención, tratamiento o cuadro de base del caso. Debe incluir objetivo de la intervención o actividad a realizar.

- Antecedentes del caso: información demográfica y cronológica de eventos importantes relacionados con el tema estudiado, antecedentes familiares o personales de relevancia, signos y síntomas (si corresponde), antecedentes antropométricos, antecedentes clínicos y bioquímicos, caracterización de la dieta, diagnóstico nutricional integrado, estilo de vida y otra información clínica o similar relevante.

- Evaluación diagnóstica: justificación de la determinación de intervención o procedimiento dietético-nutricional a realizar con la o las personas participantes.

- Intervención terapéutica: descripción de la intervención realizada en el paciente atendido.

- Seguimiento de intervención: narración de la o las actividades realizadas (cómo, cuándo y dónde), tolerancia a la intervención y posibles aspectos adversos, e identificación del instrumento para recoger y evaluar la intervención.

- Resultados: identificación final del proceso de intervención o de la situación clínica del caso.

- Discusión: confrontación de los resultados propios con la evidencia teórica del tema tratado.

- Conclusiones: comentario breve acerca del cumplimiento de las metas de la intervención o seguimiento del caso.

- Consentimiento informado: identificación del uso de instrumento y su aplicación.

- Agradecimientos: si corresponde, sin identificar al paciente o pacientes que participaron en la intervención o seguimiento del caso.

\section{Fase 6: difusión académica de los resultados}

Una vez entregado y evaluado el escrito por parte del equipo docente, se realizó una presentación oral y pública de la intervención y de sus resultados, destinada a la comunidad universitaria, en especial para estudiantes de la carrera de Nutrición y Dietética, utilizando como metodología el formato póster.

\section{Fase 7: producto final}

La actividad concluyó en un estudio de caso escrito, tipo artículo científico, en el que se describe y analiza la intervención nutricional a través del uso de la dietoterapia en una persona mayor con patología asociada.

\section{Evaluación de la intervención}

Uno de los propósitos de este estudio era obtener la opinión de los beneficiarios de esta estrategia educativa, lo que se consiguió mediante cuestionarios de preguntas abiertas, aplicados al inicio (véase fase 3 ) y al final de la intervención:

- ¿Qué sabía acerca de los estudios de casos como artículo científico antes de empezar este curso?

- ¿Cuántas veces había leído un estudio de caso? Si su respuesta es negativa, ¿por qué?

- ¿Qué tareas son las que más le han costado en todo este proceso de trabajar para el desarrollo del estudio de caso?

- ¿Le resultó complejo seleccionar a la persona o personas que participarían en su trabajo académico y fijar un objetivo para el desarrollo de su estudio de caso? ¿Por qué?

- ¿Le resultó difícil diseñar una intervención y su seguimiento en el trabajo académico del estudio de caso?

- ¿Cree que el estudio de caso le permitirá adquirir nuevas habilidades? ¿Por qué?

Previamente al primer cuestionario, se solicitó la firma del consentimiento informado por parte de todos los estudiantes del grupo, actividad que refuerza los aspectos éticos de esta intervención.

Los resultados se analizaron mediante la técnica de análisis del contenido y a través de la técnica de triangulación de informante. Las respuestas de cada sujeto fueron codificadas, analizadas, recodificadas e interpretadas para obtener las reflexiones finales por parte del equipo ejecutor. En la actividad de análisis participó también un sociólogo con experiencia en metodología cualitativa. 


\section{Resultados}

Según lo informado en los cuestionarios, los participantes reconocen haber adquirido, en el transcurso de la actividad, variadas habilidades: el fortalecimiento del aprendizaje a través del avance de las etapas del estudio de caso, el incremento de sus capacidades de redacción y vocabulario técnico, el manejo de pacientes mediante la intervención dietético-terapéutica y el desarrollo de habilidades comunicativas científicas.

Si se compara lo reportado en ambos cuestionarios sobre las nociones básicas que tienen los estudiantes en torno al estudio de caso, se observa que antes de realizar la intervención, en la cual se les explicó en qué consistía un estudio de caso, vinculaban éste con alguna patología, con su manejo o identificación. Sin embargo, una vez finalizada la actividad, logran profundizar más detalladamente en los aspectos requeridos para informar acerca del caso, como se ejemplifica en la siguiente cita: 'una presentación escrita con antecedentes de un paciente, aplicando una intervención acorde a sus necesidades, y finalmente comparando esto con la bibliografía' (estudiante 6).

Los conocimientos previos en torno al estudio de caso son confrontados con nuevos aspectos que se han ido adquiriendo y que, en cierta manera, son percibidos como enriquecedores para su aprendizaje por parte de los participantes, considerando la posibilidad de abordar el estudio de caso como artículo científico y las proyecciones que esto permite: 'antes de comenzar el curso tenía conocimiento sobre los casos clínicos, pero la actividad me ayudó a desarrollar uno y a entender mucho más su estructura y realización' (estudiante 4).

Para el grupo de estudiantes participantes, una aportación en su aprendizaje fue la incorporación del proceso de búsqueda de evidencias realizado al finalizar su escrito porque debieron desarrollar habilidades en la utilización de diversas bases de datos. Indican, además, que se incrementaron las habilidades de redacción científica en tanto conocieron nuevas exigencias del formato escrito del género discursivo denominado 'estudio de caso'. Esta percepción por parte de los estudiantes evidencia el carácter trascendente de la redacción en todo el proceso, puesto que la escritura se presenta, en primera instancia, como la mayor dificultad, pero también se convirtió en su mayor aprendizaje. A juicio de los participantes, tal habilidad les posiciona de manera ventajosa en cuanto a competitividad profesional, ya que fortalece el dominio del lenguaje escrito y la rigurosidad científica y, al mismo tiempo, promueve el aprendizaje de competencias disciplinares con la práctica en ambiente real. En definitiva, el estudio de caso se convierte en una gran herramienta didáctica y en factor de cambio en el manejo dietético-terapéutico de los pacientes, interiorizando y proyectando a su vez tales habilidades hacia el nuevo mundo profesional que se les avecina: 'nos permitió generar mejor redacción y cómo tratar a una persona como nuestro paciente' (estudiante 9).

Los cuestionarios permitieron identificar que los estudiantes declaran grandes dificultades en la realización del apartado 'discusión' del estudio de caso; tal opinión se basa principalmente en la rigurosidad que se requiere para su elaboración: 'la discusión [...] debido a que hay que realizar una lectura detenida y detallada de los otros casos con los que se comparan nuestros resultados' (estudiante 3).

\section{Discusión}

La necesidad de identificar estrategias que favorezcan la construcción del aprendizaje en los estudiantes ha permitido reconocer métodos que pueden ser de utilidad más allá de su fin tradicional. Si bien clásicamente el estudio de caso se ha señalado como una de las formas de realizar difusión académica de intervenciones en salud o presentación de alguna patología [3], también puede destinarse a la formación profesional de estudiantes del área de salud. Nuestra experiencia se basa en su uso para la formación disciplinar de estudiantes de nutrición y dietética, con etapas en su desarrollo que son identificadas por los beneficiarios como aportaciones o limitaciones para su experiencia académica.

Mejía y García [22] utilizaron el estudio de caso como parte de una estrategia de aprendizaje para estudiantes de la Facultad de Medicina de la Universidad El Bosque (Colombia). Al igual que en nuestra experiencia, sus conclusiones mencionan el refuerzo de la participación activa en la formación de los estudiantes, además de permitir el análisis reflexivo que mejore la toma de decisiones terapéuticas, fortaleciendo habilidades sociales como la comunicación y la escucha activa.

En nuestro estudio, la búsqueda de referencias en bases de datos es percibida por los estudiantes como una fortaleza, ya que se puede interpretar como un progreso para su formación profesional. Si bien en el análisis de la intervención educativa no se profundizó en la forma en que los estudiantes utilizan esta herramienta de consulta, la opinión de los encuestados coincide con los resultados de Guerra et al [23] con estudiantes de enfermería de la 
Universidad de Sevilla (España), que con posterioridad a la formación en alfabetización digital, incrementan los conocimientos en búsqueda de evidencias; esto fortaleció finalmente el aprendizaje reflexivo y significativo de los estudiantes.

En cuanto a las mejoras en competencias de redacción científica promovidas por el estudio de caso, Franco y Rodríguez [24] refieren que la publicación de los resultados de una intervención o investigación debe comenzar durante el pregrado, con contenidos técnicos, éticos y de rigor científico. En este sentido, nuestro grupo identifica también como una fortaleza la oportunidad que el estudio de caso les brindó para desarrollar sus habilidades en escritura científica, acrecentando de este modo su formación profesional. Esto se corresponde con los resultados encontrados por Bendezú et al [25] en estudiantes del área médica, quienes mencionan que el nivel de capacitación recibido en su etapa de formación profesional en esta área es evaluado como regular o deficiente. Por otra parte, Alfonso et al [26] identifican cierta dificultad en la redacción de resultados de investigaciones realizadas por estudiantes de educación superior, desde errores ortográficos hasta dificultades metodológicas o de integración de conclusiones. Los estudiantes del presente estudio no mencionan esta limitación en los cuestionarios, pero el equipo docente logra identificar debilidades de este tipo en los escritos de los participantes.

Otra instancia aludida en el proceso de desarrollo del estudio de caso es la realización de la discusión. Al respecto, Tirado et al [8] y Blanco et al [27] coinciden con nuestros encuestados en que uno de los aspectos complejos de escribir corresponde a este apartado; efectivamente, se requiere mucho rigor para revisar el caso a la luz de lo comunicado en la bibliografía científica previa. De estos resultados se deriva que, en esta zona del pensamiento y escritura científica, los docentes tenemos más que aportar durante el transcurso del proceso de enseñanza-aprendizaje.

Si bien no fue el tema central observado en esta estrategia didáctica, la intervención dietético-terapéutica en las personas mayores fue también valorada positivamente por los estudiantes. Los beneficios de actividades educativas en salud han sido comunicados por diversos autores en intervenciones desde distintas disciplinas, como los trabajos realizados por Fernández y Manrique [28] en enfermería, Díaz e Hidalgo [29] en odontología u Oliva et al [30] en nutrición, entre otros. En nuestra experiencia, los estudiantes citan beneficios en la intervención realizada que incorporan desde apren- dizajes disciplinares en el manejo terapéutico de una persona mayor a través de la dieta, hasta la capacidad de comprender e intervenir en un contexto real a una persona mayor con patología asociada.

Como una limitación de nuestro estudio estuvo el hecho de no profundizar de forma exhaustiva en la experiencia que significó la presentación oral, actividad realizada al finalizar la intervención. Al respecto, Ferrero [31] señala que las ponencias orales mejoran las habilidades comunicativas al reducir el temor a hablar en público, y también otras competencias, como las auditivas. Asimismo, la expresión escrita también se ve reforzada toda vez que, antes de la presentación oral, existe una planificación y ordenación de ideas.

Al ejecutar una atención nutricional en pacientes reales, los estudiantes se ven enfrentados a utilizar la aplicación de competencias aprendidas en su formación profesional. Este aspecto va estrechamente ligado a la adquisición de nuevas habilidades comunicativas y de atención con el paciente, ya que en la práctica deben recurrir a estas herramientas con el fin de obtener la cercanía y confianza necesarias para realizar una buena intervención. Esa aproximación al usuario beneficiado es utilizada por los estudiantes en las visitas domiciliarias correspondientes, aprendiendo y fortaleciendo a la vez el trato directo con el paciente. Por último, los participantes de este estudio aseguran haber obtenido mayor experiencia y la posibilidad de conocer una patología desde el punto de vista de las personas atendidas, más allá de lo aprendido, hasta el momento, de manera teórica. Esta opinión posiciona la experiencia como una adecuada herramienta de aprendizaje práctico de las competencias adquiridas en años de formación académica.

En conclusión, el estudio de caso, conocido por todo el equipo médico en su formación disciplinar o en la práctica diaria, puede constituir una estrategia educativa integral porque, además de promover el aprendizaje disciplinar activo de los estudiantes en alguna área de las ciencias de la salud -como el diseño e implementación de un plan de manejo dietético-terapéutico en adultos mayores en este estudio-, puede repercutir también en la formación de otras competencias comunicativas orales y escritas necesarias para la formación académica y científica de los profesionales de la salud.

\footnotetext{
Bibliografía

1. Martínez G, Noreña A, Martínez J, Ortiz R. Methodology review for writing and publishing case report: applications on the nutrition field. Nutr Hosp 2015; 32: 1894-908.
} 
2. Romani F. Case report and case series: an approach for undergraduate. CIMEL Ciencia e Investigación Médica Estudiantil Latinoamericana 2010; 15: 46-51.

3. Villanueva I. Cómo redactar un caso clínico. Acta Ortop Mex 2009; 23: 315-6.

4. López D, Torres A. Recommendations for drafting, designing and structuring a publication of clinical case. Rev Esp Med Quir 2014; 19: 229-35.

5. Vega O. Practical guide to write and publish clinical cases. Revista Cuadernos 2017; 58: 47-51.

6. Vega J. How to write a case report for publication. A practical guide. Rev Med Chile 2015; 143: 499-505.

7. Delgado M. New guidelines for case reports. RFCS Rev Fac Cien Sal Univ Cauca 2013; 15: 47-50.

8. Tirado G, Hueso C, Cuevas M, Montoya R, Bonill C, Schmidt J. How to write clinical cases studies in nursing using the NANDA, NOC, NIC taxonomies. Index Enferm 2011; 20: 111-5.

9. Burdiles G. Clinical case study genre: rhetorical organization of the macromove case report in Chilean medical journals. Rev Signos 2016; 49): 192-216.

10. Martínez J. Styles of presentation of clinical cases and other interesting cases. Rev Med Electrón 2017; 39: 381-3.

11. Ordóñez L, Fernández Y. Necessity to unify approaches on the presentation of clinical cases. Rev Med Electrón 2017; 39: 378-80.

12. Margalef L, Pareja N. A one-way path: active learning methodological strategies. Revista Interuniversitaria de Formación del Profesorado 2008; 22: 47-62.

13. Montes N, Machado E. Estrategias docentes y métodos de enseñanza-aprendizaje en la educación superior. Rev Hum Med 2011; 11: 475-88.

14. Ventura A. Estilos y estrategias de aprendizaje relacionados con el logro académico en estudiantes universitarios. Pensam Psicol 2013; 11: 115-29.

15. Ventura A. El ajuste instructivo entre estilos de aprendizaje y enseñanza en la universidad. Rev Psicol 2013; 31: 265-86.

16. Loret de Mola J. Estilos y estrategias de aprendizaje en el rendimiento académico de estudiantes de la Universidad Peruana Los Andes de Huancayo-Perú. Revista Estilos de Aprendizaje 2011; 8: 1-37.

17. Acevedo D, Durán M, Alvis A. Identificación de estrategias de aprendizaje de estudiantes de ingeniería de alimentos en los cursos Balance de Materia, Transporte de Fluidos y Operaciones Unitarias. Form Univ 2015; 8: 31-8.
18. Juárez C, Rodríguez G, Escoto M, Luna E. Relación de los estilos y estrategias de aprendizaje con el rendimiento académico en estudiantes universitarios. Revista Estilos de Aprendizaje 2016; 9: 268-88.

19. Pool W, Martínez J. Autoeficacia y uso de estrategias para el aprendizaje autorregulado en estudiantes universitarios. REDIE 2013; 15: 21-36.

20. Monroy F, Hernández F. Factores que influyen en los enfoques de aprendizaje universitarios. Una revisión sistemática. Educación XXI 2014; 17: 105-24.

21. Burdiles G. Descripción de la organización retórica del género caso clínico de la medicina a partir del corpus CCM-2009 [tesis doctoral]. Valparaíso: Pontificia Universidad Católica de Valparaíso; 2012.

22. Mejía O, García G. Teaching techniques: clinical case approach to the use of video as a tool to support. Revista de la Universidad Industrial de Santander Salud 2013; 45: 29-38.

23. Guerra M, Lima M, Zambrano E, Lima J. Is information literacy learning effective for nursing students? Enferm Glob 2014; 13: 90-102

24. Franco C, Rodríguez A. Common mistakes in student scientific writing. Gac Méd Caracas 2010; 118: 69-73.

25. Bendezú G, Hurtado S, Madina C, Aguilar P. Appreciation of research training and scientific publication in college students. Invest Educ Médica 2015; 4: 50-1.

26. Alfonso A, Zamora L, Águila O. Pharmacology and medical computing interdisciplinary work for the improvement of the students' investigations. Edumecentro 2016; 8: 121-33.

27. Blanco M, Valdez R, Blanco O. Methodology-scientific evaluation of the clinical case presentation articles published in the Revista Habanera de Ciencias Médicas. 2010-2015. Rev Haban Cienc Méd 2016; 15: 536-50.

28. Fernández A, Manrique F. Effect of the educational intervention in the self-care agency for elderly hypertensive in BoyacáColombia, Sudamérica. Cienc Enferm 2010; 16: 83-97.

29. Díaz Y, Hidalgo L. Educative intervention on oral health in primary school teachers. Rev Med Electrón 2011; 33: 52-9.

30. Oliva R, Tours M, Gil B, Longo G, Pereira J, García P. Impact of a brief educational intervention about nutrition and healthy lifestyles to school students given by a healthcare provide. Nutr Hosp 2013; 28: 1567-73.

31. Ferrero L. Oral presentations in the classes. Publicaciones Didácticas 2012; 31: 19-21. 
